\title{
Clinical Response of Enhanced External Counter Pulsation (EECP) Among Bangladeshi Ischemic Heart Disease Patients
}

\author{
KMHS SIRAJUL HAQUE ${ }^{1}$, MD. JABED IQBAL ${ }^{2}$, MD. MAHMUDUR RAHMAN SIDDIQUI ${ }^{3}$, KHONDAKER SYEDUS \\ SALEHIN ${ }^{4}$
}

${ }^{1}$ Department of Cardiology, Bangabandhu Sheikh Mujib Medical University, Shahbag, Dhaka. ${ }^{2}$ The Medical Centre, House - 84, Road - 7/A, Dhanmondi, Dhaka. ${ }^{3}$ Department of Medicine, Dhaka Medical College, Dhaka. ${ }^{4}$ Department of Medicine, Bangabandhu Sheikh Mujib Medical University, Shahbag, Dhaka

Address of correspondence: Dr. Md. Jabed Iqbal, The Medical Centre, House \# 84, Road \# 7/A, Dhanmondi, Dhaka. E-mail:dr.jabed.iqbal@gmail.com.

\begin{abstract}
:
EECP is used to stabilize the coronary circulation in patients with severe coronary artery disease when maximal medical therapy and/or invasive procedures have proven inadequate. This prospective observational study was done for a period of 12 months. 40 patients with angina and angiographically proven coronary artery disease, who met the inclusion and exclusion criteria was enrolled in this study. Among 40 enrolled patients 30 (70\%) patients completed their treatment. All the patients were in between 42 to 80 years age group. All patients required sublingual nitrate before started EECP treatment. But after completion of treatment, $75 \%$ patient required no nitrates. Who completed full course, $100 \%$ were improved their angina symptoms from class II, III, IV to class I. Overall $80 \%$ patients improved their quality of life. Those completed full course of treatment $100 \%$ patients improved their quality of life. EECP Heart therapy is now world-wide accepted alternative treatment option for those patients, who are not fit for surgery or any kind of interventional procedure and those developed heart failure.
\end{abstract}

Key Words: Enhanced External Counter Pulsation (EECP), Ischemic Heart Disease

\section{Introduction:}

Enhanced External Counterpulsation (EECP) is a noninvasive mode of treatment to improve myocardial perfusion and cardiac output, thereby reducing symptoms of angina and heart failure. ${ }^{1}$ It was given on outpatient basis. EECP is used to stabilize the coronary circulation in patients with severe coronary artery disease when maximal medical therapy and/or invasive procedures have proven inadequate. ${ }^{1}$ Improved technology in percutaneous transluminal coronary angioplasty (PTCA) and coronary artery bypass graft (CABG) has made it possible to successfully treat patients with chronic angina pectoris. However, a large number of patients remain symptomatic in spite of PTCA or CABG, continue to be ineffective or associated with an unacceptable risk of complications. There is an increasingly large population of patients who have persistent residual anginal symptoms and they remain severely restricted physically. ${ }^{2}$ Following bypass surgery, $75 \%$ of patients are symptom free from ischemic events for 5 years or more, and $50 \%$ remain so after 10 years or more. ${ }^{3} \mathrm{EECP}$ has been shown to be effective in these group of patient. ${ }^{4,5}$ Complete resolution of ischemic perfusion defects was achieved in $67 \%$, partial reperfusion with decrease in size of defects was noted in $11 \%$, and no change was seen in $22 \%$ of patients. ${ }^{5}$
In 1953, Kantrowitz brothers first described diastolic augmentation of coronary arteries at the Massachuetts Institute of Technology. ${ }^{6}$ Sarnof demonstrated that left ventricular work and myocardial oxygen consumption depend directly on end diastolic pressure. ${ }^{6}$ William Birtwell developed an early intravascular arterial pulsator, which drew off blood during systole and returned it during diastole. Richard Gorlin named this technique counterpulsation. ${ }^{6}$ Lawson et al, at the State University of New York, Stony Brook, undertook a number of open label studies with the enhanced system, EECP, between 1989 and 1998 using both objective and subjective end points. ${ }^{5,7-9}$ In 1999, Arora et al, reported results of the first double-blind randomized placebo-controlled multicenter trial (MUST-EECP). ${ }^{10}$ Since then, EECP therapy has emerged as a safe, highly beneficial, low cost, non-invasive treatment of refractory angina patients and now for heart failure patients as well. Life of the ischemic heart disease patients has been prolonged due to modern treatment and after a period they report with recurrent chest pain i.e. refractory angina and refractory heart failure. Few study related with EECP is available in the country. An endeavour has been made to undertake a prospective study to know the clinical response of EECP in Bangladeshi patients. 


\section{Aims and Objectives:}

To find out improvement of chest pain and breathlessness, if any after EECP therapy in patient with coronary artery disease.

\section{Materials and Methods:}

It was a prospective observational study. The study was done in The Medical Centre, Dhaka, Bangladesh for a period of 12 months (January 2010-December 2010). The study was performed among the patients who were subjected EECP Therapy in The Medical Centre, Dhaka with clinical features and angiographic findings of coronary artery disease. A total of 40 patients with angiographically proven coronary artery disease, who met the inclusion and exclusion criteria were enrolled in this study. All patients were suffering from angina and documented coronary artery disease (CAD). Angina was graded according to Canadian Cardiovascular Society (CCS) angina classification. Total 35 hours (1 hour daily) of therapy was given with standard pressure of $260 \pm 20 \mathrm{~mm}$ Hg. Treatment effect was determined by clinical evaluation, relief of angina, use of sublingual nitrates, and physical wellbeing and compared with the patients who completed full course of treatment (treatment-complete group) and who were not completed their full treatment (treatmentincomplete group). Quality of life was assessed by WHO performance status score. Statistical analysis was conducted on SPSS 16.0 for windows software.

\section{Inclusion criteria:}

- $\quad$ Angiographically proven coronary artery disease.

- $\quad$ Refectory angina after CABG or PTCA or Medical treatment.

- $\quad$ Patient unwilling to do any interventional procedure.

- Anatomy of coronary artery not suitable for intervention like CABG/PCI.

- $\quad$ Patient suffering from medical diseases, for which intervention procedures are contraindicated.

- $\quad$ CABG not possible due to low ejection fraction (EF).

\section{Exclusion criteria:}

- $\quad$ Coagulopathy with an INR of prothrombin time 2.5.

- Arrhythmias that may interfere with triggering of EECP system

- Within 2 weeks after cardiac catheterization or arterial puncture.

- $\quad$ Decompensated heart failure.

- Moderate to severe aortic insufficiency.

- $\quad$ Severe peripheral arterial disease.

- $\quad$ Severe hypertension 180/110 mm Hg.

- Aortic aneurysm or dissection.

- $\quad$ Pregnancy.
- Venous disease (phlebitis, varicose veins, stasis ulcers, prior or current deep vein thrombosis or pulmonary embolism).

- $\quad$ Severe chronic obstructive pulmonary disease.

- $\quad$ Severe pulmonary hypertension (PASP >50 mmHg).

\section{Results:}

In this study, among 40 patients 34 (85\%) were male and 6 (15\%) were female patients and all patients were in between 42 to 80 years (Mean 58.82) age group. All patients were suffering from angina pectoris and 23 (58\%) had previous history of myocardial infarction and 10 (25\%) of them had IHD with heart failure. Most of the patients ( $n=31,77.5 \%)$ had CCS class III and class IV angina. 25 (62.5\%) patients had triple vessel disease, 9 (22.5\%) had double vessel disease and 5 (12.5\%) had single vessel disease and 1 (2.5\%) was suffering from syndrome X (figure-I). 30 (75\%) patients completed there full course of treatment. Among those who were not completed ( $n=10,25 \%)$, two patients were suffering from unstable angina, one developed haemorrhoidal bleeding (which was not disclosed earlier), one developed respiratory tract infection, one developed acute exacerbation of peptic ulcer disease, three were not continued treatment due to economical problem and two had transportation problem. After EECP therapy among treatment completed group all 30 patients (100\%) improved to CCS class I from class III and IV and 5(50\%) out of 10 of treatment incomplete group patients also improved to CCS class I (figure-IV). All the treatment completed patients improve their quality of life. Before treatment 39 (97.5\%) patients used sub lingual $(\mathrm{S} / \mathrm{L})$ but after completion of treatment overall 30 (75\%) patients did not require S/L nitrate and another 4 (10\%) patients required occasional nitrate use (figure-VII).

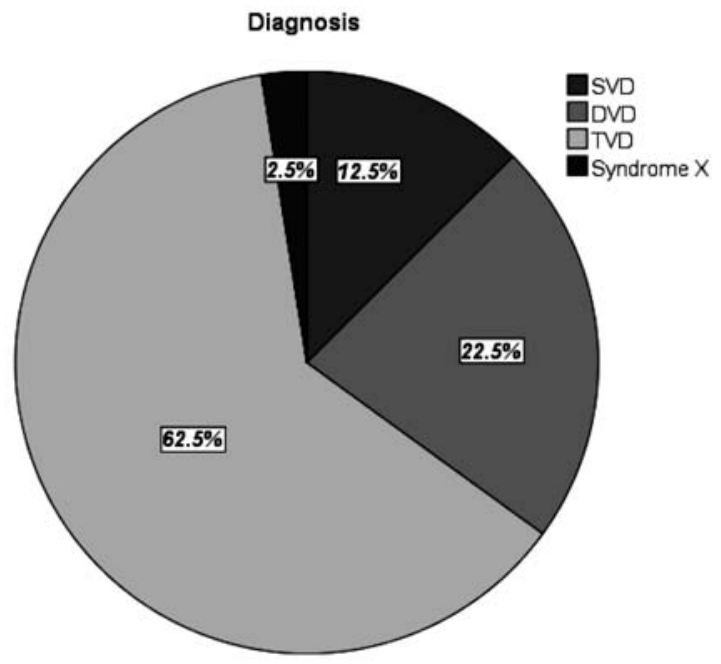

Fig.-I: Diagnosis of the patient ( $N=40$, SVD: single vessel disease, DVD: double vessel disease, TVD: triple vessel disease) 


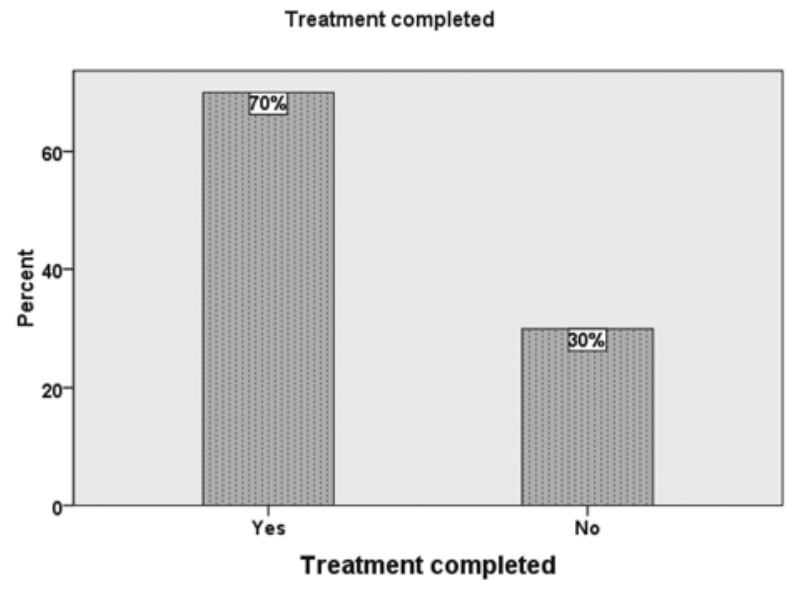

Fig.-II: Patients completed EECP Therapy $(N=40)$

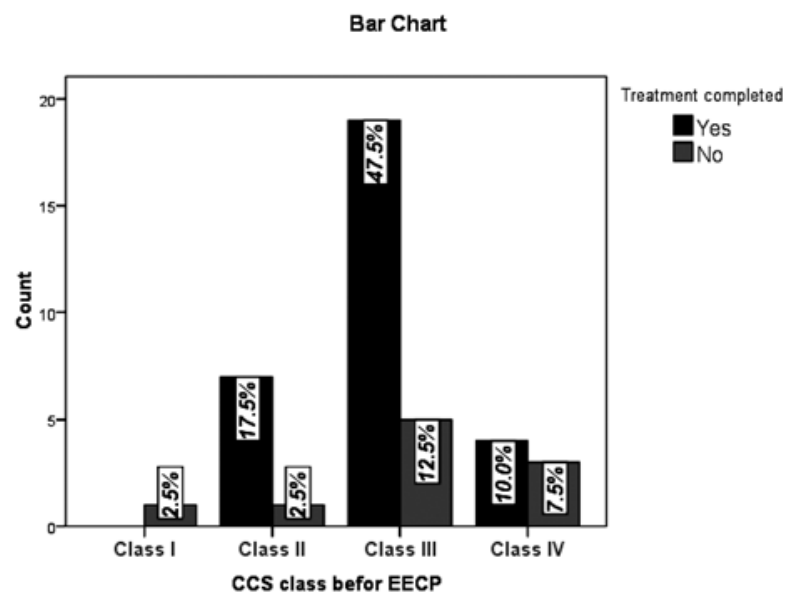

Fig.-III: Distribution on CCS Class before EECP Therapy $(N=40)$

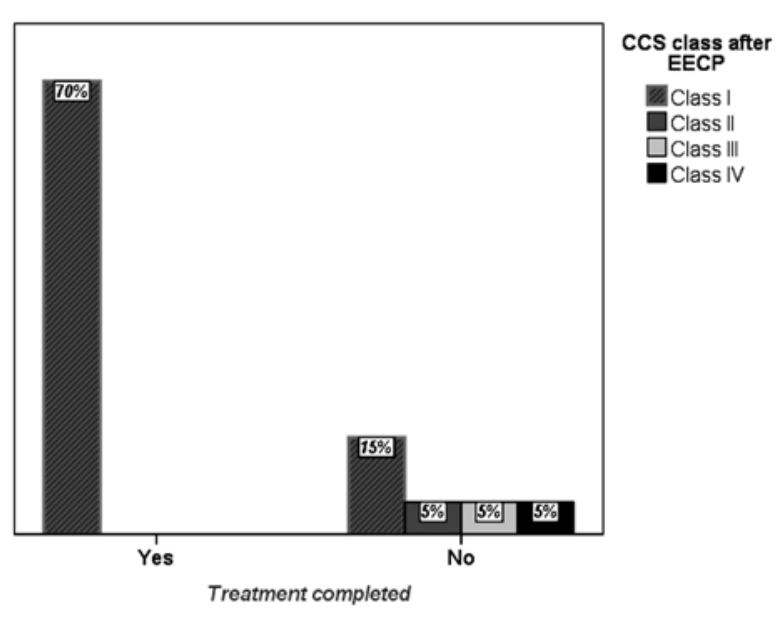

Fig.-IV: Distribution on CCS Class after EECP Therapy $(N=40)$

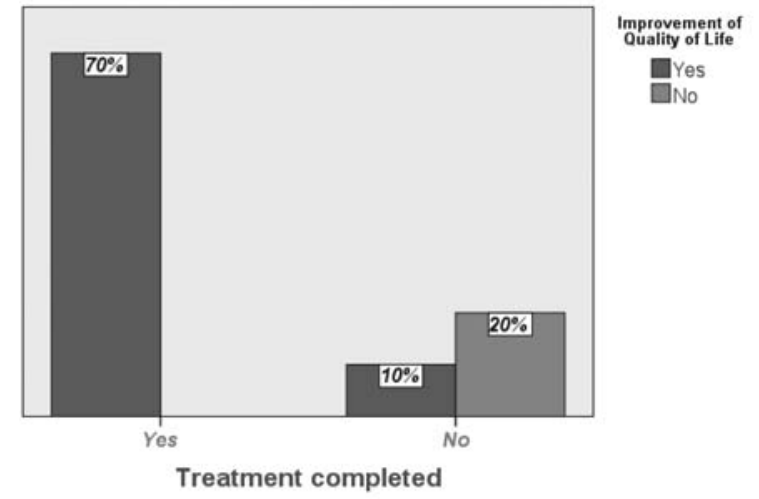

Fig.-V: Distribution of the patients on Improvement of Quality of Life after EECP therapy $(N=40)$

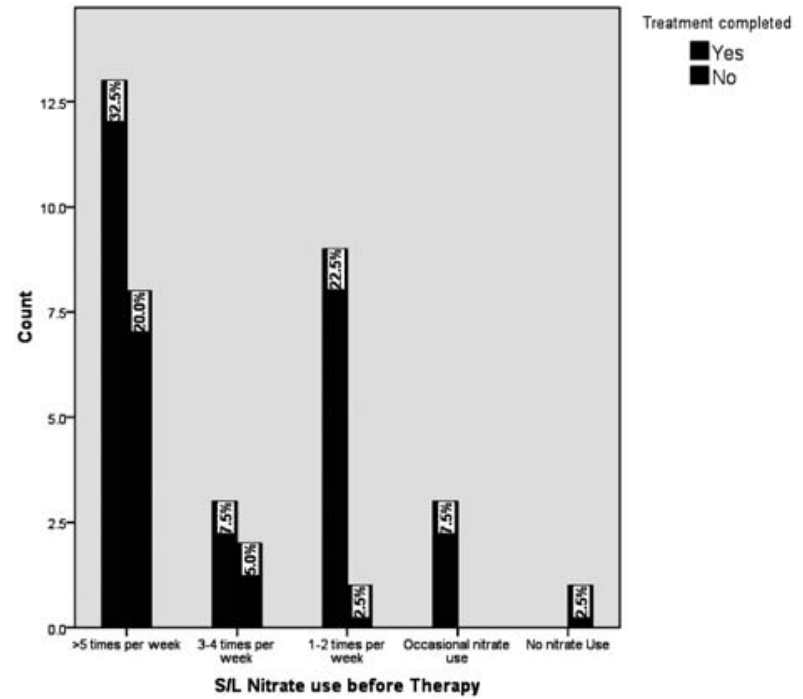

Fig.-VI: S/L Nitrate use before EECP $(N=40)$

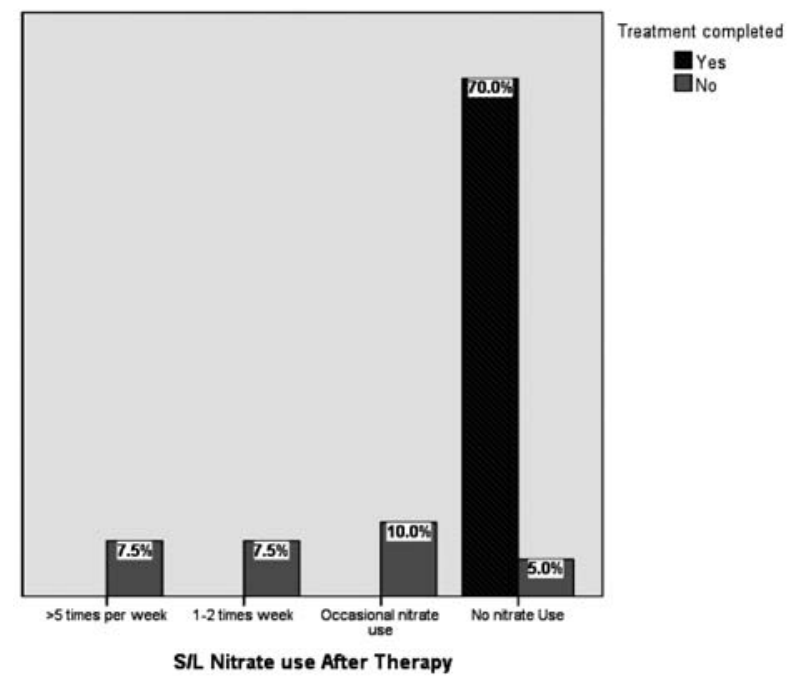

Fig.-VII: S/L Nitrate use after EECP $(N=40)$ 


\section{Discussion:}

In this study, it has been found that those patients who completed full 35 hours treatment, improves significantly. Among 40 enrolled patients 30 (70\%) patients completed their treatment. All the patients were in between 42 to 80 years age group. Figure-I shows that, maximum 25 (62.5\%) patients had TVD, 9 (22.5\%) had DVD, 5(12.5\%) had SVD and only $1(2.5 \%)$ had syndrome X disease. 39(97.5\%) patients required S/L nitrate before starting of EECP treatment. Before treatment 21 (52.5\%) patients used sub lingual (S/L) more than 5 times per week but after treatment overall 30 (75\%) patients did not use S/L nitrate (figureVII). Those who were not completed full course of treatment showed improved significantly. Patients who were with refractory angina improved significantly. This result is close to the finding of MUST-EECP trial. ${ }^{10}$ Before EECP therapy most of the treatment-completed (47.5\%) and treatment not-completed (12.5\%) patients were in CCS class III group (figure-III). Total 24 (60\%) patients had CCS III angina. After EECP therapy among treatment completed group all patients (100\%) improve to CCS class I from class II, III, IV (figure-IV). Those were not completed full course but completed $>15$ hours, 5 (50\%) of them improves angina class. External counterpulsation has been shown to reduce the frequency and severity of anginal attacks in patients with symptomatic coronary artery disease. ${ }^{11}$ Among 40 patients overall 35 (80\%) patients improved their quality of life and those completed full course of treatment $100 \%$ patients improved their quality of life. These results were consistent with other international studies like PEECH trial. ${ }^{12}$ Many clinical trials in the last two decades have shown enhanced external counterpulsation (EECP) therapy to be safe and effective for patients with refractory angina with clinical response rate averaging $70 \%$ to $80 \%$ that is sustained up to 5 years. ${ }^{13}$ Enhanced external counterpulsation therapy is a comparatively easy and useful out-patient procedure providing acute and long-term relief of angina symptoms and improved quality of life among a group of patients with symptomatic ischemic heart disease with or without congestive heart failure. ${ }^{14}$

\section{Conclusion:}

This study shows that EECP therapy seems to be beneficial and alternative treatment of the refractory angina. EECP Heart therapy is now world-wide accepted alternative treatment option for those patients, who are not fit for surgery or any kind of interventional procedure and those developed heart failure. It is also a good option for refractory angina, who are not willing to do any interventional procedure. In Bangladesh this therapy is introduced recently, so more clinical experience will be obtained from further study in near future.

\section{Acknowledgment:}

All Patients and the authority of The Medical Centre, Dhanmondi, Dhaka, Bangladesh.

\section{References:}

1. John ES, Robert B, Joseph CH. The emerging role of enhanced external counterpulsation in cardiovascular disease management. The Journal of cardiovascular management; Sept-Oct 1998:23-29.

2. Tony MC. Enhanced external counterpulsation. ACC educational highlights/Summer; 1998:1-4.

3. Foster ED, Reoperation for coronary artery disease. Circulation. 1985;72 (suppl):57-64.

4. Zheng ZS, Li TM, Kambie H, Chen GH, et al: Sequential external counterpulasation (SECP) in China. Trans Am Soc Artif Organs 1983; 29: 599-603.

5. Lawson WE, Hui JCK, Soroff HS, et al: Efficacy of external counterpulsation in the treatment of angina pectoris. Am J Cardiol 1992; 70:859-62.

6. Soran O, Crawford LE, Schneider VM, Feldman AM. Enhanced external counterpulsation in the management of patients with cardiovascular disease. Clin Cardiol 1999; 22:173-8.

7. Lowson WE, Hui JC, Zheng ZS, et al; Improved exercise tolerance following enhanced external couterpulsation: cardiac or peripheral effect? Cardiology 1996; 87:271-5.

8. Lowson WE, Hui JCK, Zheng ZS, et al;Can angiographic findings predict which coronary patients will benefit from enhanced external counterpulsation? Am J Cardiol. 1996; 77: 1107-9.

9. Lowson WE, Hui JCK, Guo T, et al; Prior revascularization increases the effectiveness of enhanced external counterpulsation. Clin Cardiol. 1998; 21: 841-4.

10. Arora RR, Chou TM, Jain D, et al. "The multicenter study of enhanced external counterpulsation (MUST-EECP): effect of ECP on exercise-induced myocardial ischemia and anginal episodes”. J. Am. Coll. Cardiol 1999; 33(7):1833-40.

11. Hunt SA, Abraham WT, Chin MH et al. 2009 Focused Update Incorporated Into the ACC/AHA 2005 Guidelines for the Diagnosis and Management of Heart Failure in Adults: A Report of the American College of Cardiology Foundation/American Heart Association Task Force on Practice Guidelines Developed in Collaboration With the International Society for Heart and Lung Transplantation. J. Am. Coll. Cardiol. 2009; 53(15): e39-e40.

12. Feldman AM, Silver MA, Francis GS, De Lame PA, Parmley WW. "Treating heart failure with external counterpulsation (ECP): design of the Prospective Evaluation of ECP in Heart Failure (PEECH) trial”. J. Card. Fail April 2005; 11(3):240-5.

13. Manchanda A, Soran O. ”Enhanced external counterpulsation and future directions: step beyond medical management for patients with angina and heart failure”. J. Am. Coll. Cardiol 2007; 50 (16): 1523-31.

14. Siddique MA. Is Enhanced External Counterpulsation (EECP) A- 'Last Resort' Treatment of Refractory Angina? University Heart Journal 2009; 5(1):1-2. 